\title{
Statin and Post-Cardiac Surgery Atrial Fibrillation Prevention: Systematic Review and Meta-analysis
}

\author{
Federico Oliveri ${ }^{1}$, Andrea Bongiorno ${ }^{1}$, Sara Compagnoni ${ }^{1}$, Alessandro Fasolino ${ }^{1}$, Francesca \\ Gentile $^{1}$, Antonella Pepe ${ }^{1}$, and Lorenzo Tua ${ }^{1}$ \\ ${ }^{1}$ Universita degli Studi di Pavia
}

November 10, 2021

\begin{abstract}
Introduction: Postoperative atrial fibrillation (POAF) is a frequently reported complication of cardiac surgery, leading to increased in-hospital and long-term mortality rates. Many studies have suggested using statins to protect against POAF. Thus, we aim to investigate if statin pre-treatment may effectively lower the incidence of POAF. Method: We performed a systematic literature search of PubMed for potential studies between January 2006 and August 2021. Principal inclusion criteria were: randomized clinical trials study design; statin-naive patients; total study participants [?] 50 units. We used the fixed-effects model to obtain the odds ratio (OR) and 95\% confidence interval (CI) for each analyzed intervention. Results: Overall, statin pre-treatment reduced the incidence of POAF compared to placebo (OR 0.71; 95\% CI: 0.60-0.85, p-value < 0.00001). Analyzing subclasses, atorvastatin was associated with lower incidence of POAF (OR 0.54; 95\% CI: 0.41-0.70, p-value $=0.002)$, but rosuvastatin was not $($ OR 0.90; 95\% CI: 0.71-1.14, p-value $=0.38)$. Selecting studies with [?] 199 patients, results were divergent. There was not statistically significant difference between statin pre-treatment and placebo (OR 0.89 ; 95\% CI: 0.74-1.09, p-value $=0.26$ ), as well as for atorvastatin (OR 0.74; 95\% CI: 0.54-1.03, p-value $=0.08)$ and rosuvastatin (OR 0.87; 95\% CI: 0.68-1.12, p-value = 0,29). Conclusion: Statin pre-treatment before cardiac surgery is not associated with a significant reduction in POAF occurrence. Thus, based upon our results and considering possible renal complications, we discourage statin pre-treatment in preventing POAF.
\end{abstract}

\section{INTRODUCTION}

Postoperative complications frequently occur after cardiac surgery, leading to significant increases in mortality, morbidity, and $\operatorname{costs}^{\mathbf{1}}$. Notably, approximately 15 to 40 percent of patients who underwent coronary artery bypass graft surgery $(\mathrm{CABG})^{\mathbf{2 - 5}}$, as well as 38 to 50 percent after valve surgery ${ }^{\mathbf{2 , 6} \mathbf{7}}$, experience postoperative atrial fibrillation (POAF) in the early postoperative period. Several studies showed that POAF is associated with increased in-hospital and long-term mortality rates ${ }^{3-5,8-10}$ and prolonged hospitalization $6,10-12$

Pathophysiologically, POAF is believed to be related to inflammatory processes, leading to oxidative stress 13. In particular, a strict connection exists between the peak of the systemic inflammatory response after cardiac surgery and the POAF occurrence ${ }^{\mathbf{1 3}}$.

Interestingly, among the pleiotropic effects of statins are their anti-inflammatory and antioxidant properties ${ }^{\mathbf{1 4 - 1 6}}$. Thus, some clinical and experimental studies have suggested using statins to protect against atrial fibrillation ${ }^{\mathbf{1 7}}$. In keeping with these findings, some meta-analyses made from randomized clinical trials showed that preoperative statin therapy, generally introduced in the week before cardiac surgery, had been associated with a lower incidence of POAF ${ }^{\mathbf{1 8 , 1 9}}$. However, these studies pointed out significant limitations of the evidence. 
Indeed, considering the high burden of bias and the new valuable trials conducted, we performed our study to update the current knowledge regarding statins' potential role in preventing POAF.

\section{METHOD}

\subsection{Search strategy}

A systematic literature review and subsequent meta-analysis were performed according to preferred reporting Items for Systematic Reviews and Meta-Analyses (PRISMA) Guidelines ${ }^{\mathbf{2 0}}$. We systematically searched in PubMed, EMBASE, and Medline for inherent studies published starting from August 2021. In our metaanalysis, we included randomized clinical trials only. Keywords used to find the desired articles were the following: (1) statin; (2) postoperative atrial fibrillation; (3) statin and atrial fibrillation; (4) statin and postoperative atrial fibrillation. The search only included original studies on human subjects published in the English language. To include pertinent papers, two authors independently searched for additional citations from the reference list of included relevant articles.

\subsection{Study selection}

Two reviewers (F.O. - A.B.) independently performed the initial screening process to recognize all citations of potential acceptability. The inclusion criteria in our study consisted of:

randomized clinical trials (RCTs) study design;

statin-naive patients;

Rosuvastatin or Atorvastatin started no more than 21 days before cardiac surgery;

total study participants [?] 50;

studies published starting from January 2006;

Original untranslated studies that are written in English language only;

Age [?] 18 years old;

For definitive eligibility, full-text papers of recognized abstracts that were pertinent to our inclusion criteria were evaluated. The excluded papers were non-randomized clinical trials, cohort studies, case reports, letters, conference abstracts, and editorials. The Kappa statistic was utilized to evaluate the inter-rater reliability of the two reviewers ${ }^{21}$.

\subsection{Data extraction and quality assessment}




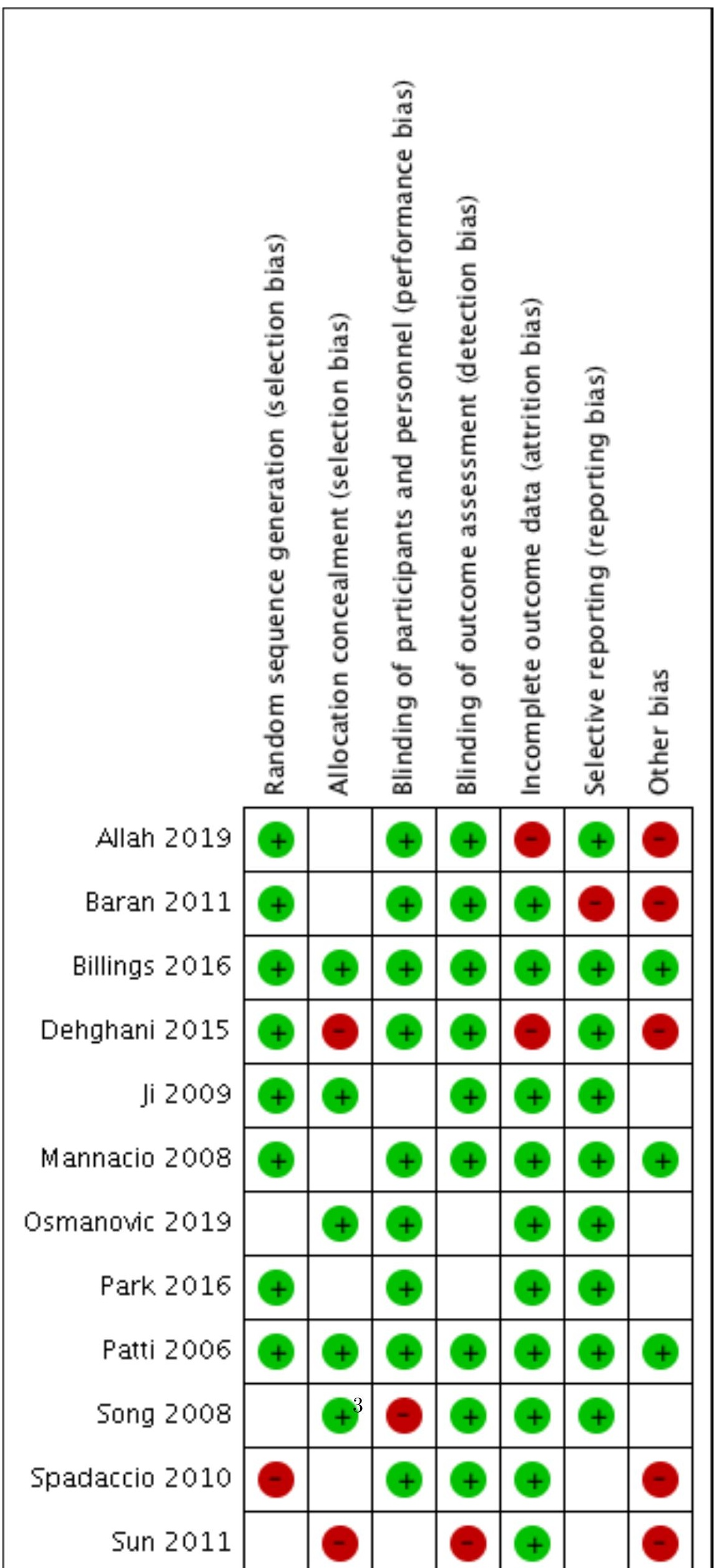


Two reviewers (F.O. - A.F.) extracted data independently using a standardized recording tool to document the study design and setting, number of study participants, year of publication, country of origin, baseline patient characteristics, participant clinical characteristics, and study outcomes.

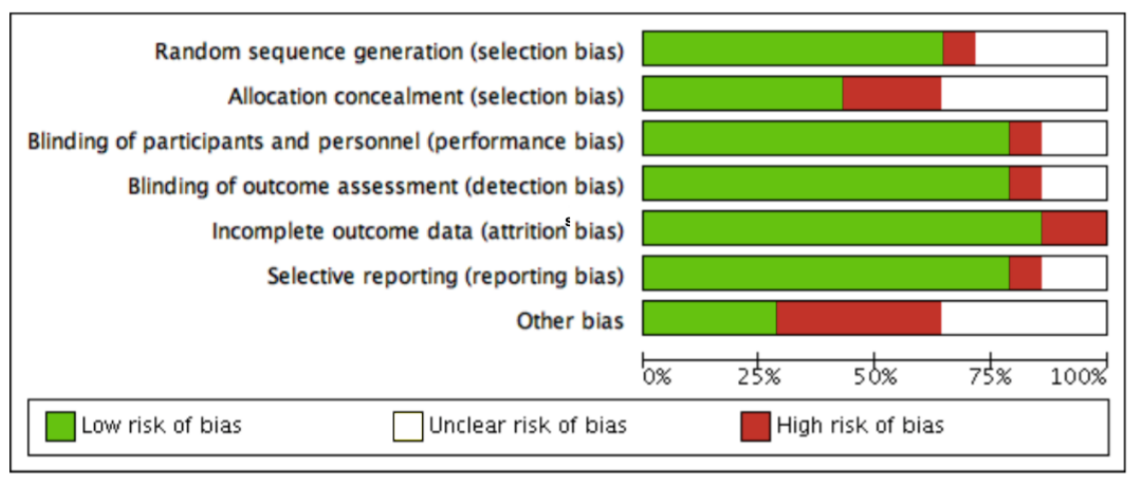

The quality of the included studies was assessed according to the Cochrane Collaboration's tool for assessing the risk of bias $^{\mathbf{2 2}}$. F.G. and A.P. assessed the risk of bias of all considered studies (including selection, performance, detection, attribution, reporting, and other biases). All discrepancies were resolved by consulting with the other authors and referring to the original articles (Figure 1 e 2) .

\subsection{Data analysis and synthesis}

We used the Review Manager software (RevMan-5) to conduct our statistical analyses. The overall odds ratio (OR), and the 95\% CI for each analyzed parameter were pooled using a fixed-effects model. Furthermore, we have drawn forest plots to evaluate the results of pooling visually. OR value $>1$ indicates an increased risk of complex POAF, OR value 1 indicates no observed association, and OR $<1$ indicates decreased risk of POAF. A two-sided P-value $<0.05$ was considered statistically significant. Furthermore, the heterogeneity of the studies results was calculated using the Higgins $\mathrm{I}^{2}$, which measures the percentage of the total variation across the included studies ${ }^{\mathbf{2 3}}$. The values of $\mathrm{I}^{2}$ lie between 0 and $100 \%$. A value of $0 \%$ indicates no heterogeneity. We classified heterogeneity in mild $\left(\mathrm{I}^{2}<25 \%\right)$, moderate $\left(25[?] \mathrm{I}^{2}<50 \%\right)$, severe $(50[?]$ $\left.\mathrm{I}^{2}<75 \%\right)$, and very severe $\left(\mathrm{I}^{2}[?] 75 \%\right)^{\mathbf{2 4}}$.

\section{RESULTS}

\subsection{Literature search}




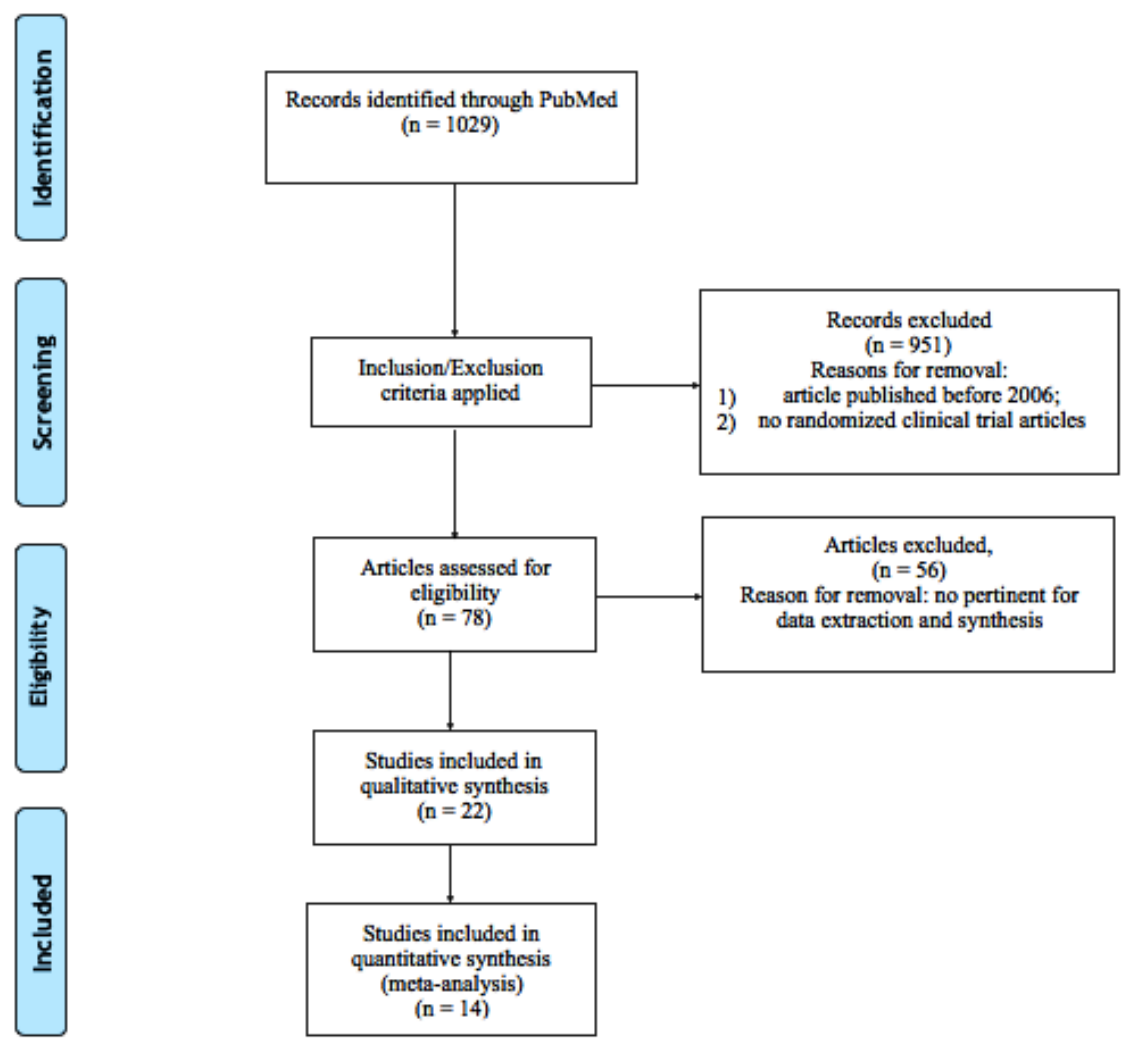

The flow diagram of studies identification and subsequent inclusion is shown in Figure 3 . 1029 citations were found by the search. The final study included a total of 78 papers, of which 22 (consisting of randomized clinical trials) were used for data extraction and synthesis for our systematic review. Fourteen articles were finally included in our meta-analysis.

\subsection{Characteristic of the included studies}

The study characteristics of the included fourteen studies ${ }^{25-38}$ are shown in Table 1 . All were randomized clinical trials and published from 2006 to 2021. The total number of individuals included in our metaanalysis was 2883 , about half of whom were pretreated with statin before cardiac surgery. The trials were conducted in 9 different countries and four continents. We evaluated the potential role of statin pretreatment in preventing POAF.

TABLE 1: CHARACTERISTICS OF THE INCLUDED STUDIES

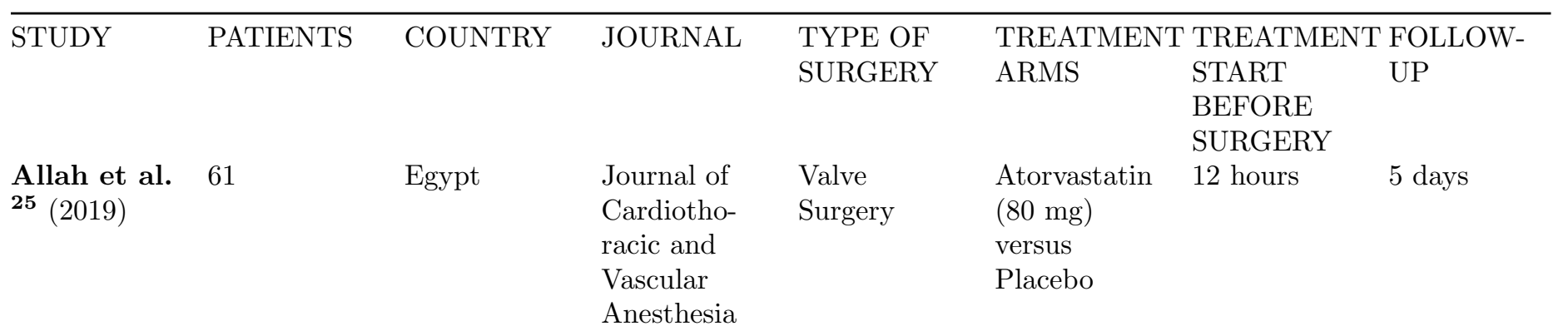




\begin{tabular}{|c|c|c|c|c|c|c|c|}
\hline $\begin{array}{l}\text { Baran et } \\
\text { al. 26 } \\
(2011)\end{array}$ & 60 & Turkey & $\begin{array}{l}\text { Stem Cell } \\
\text { Reviews and } \\
\text { Reports }\end{array}$ & CABG & $\begin{array}{l}\text { Atorvastatin } \\
(40 \mathrm{mg}) \\
\text { versus } \\
\text { Placebo }\end{array}$ & - & 30 days \\
\hline $\begin{array}{l}\text { Billings et } \\
\text { al. } 27 \\
(2016)\end{array}$ & 199 & USA & JAMA & Multiple & $\begin{array}{l}\text { Atorvastatin } \\
\text { (variable) } \\
\text { versus } \\
\text { Placebo }\end{array}$ & variable & $\begin{array}{l}\text { until } \\
\text { hospital } \\
\text { discharge }\end{array}$ \\
\hline $\begin{array}{l}\text { Dehghani } \\
\text { et al. } 28 \\
(2015)\end{array}$ & 58 & Iran & $\begin{array}{l}\text { Journal of } \\
\text { Cardiovascu- } \\
\text { lar } \\
\text { Pharmacol- } \\
\text { ogy and } \\
\text { Therapeutics }\end{array}$ & $\begin{array}{l}\text { Valve } \\
\text { Surgery }\end{array}$ & $\begin{array}{l}\text { Atorvastatin } \\
(40 \mathrm{mg}) \\
\text { versus } \\
\text { Placebo }\end{array}$ & 3 days & 5 days \\
\hline $\begin{array}{l}\text { JI et al. }{ }^{\mathbf{2 9}} \\
(2009)\end{array}$ & 140 & China & $\begin{array}{l}\text { Circulation } \\
\text { Journal }\end{array}$ & CABG & $\begin{array}{l}\text { Atorvastatin } \\
(20 \mathrm{mg}) \\
\text { versus } \\
\text { Placebo }\end{array}$ & 7 days & 13 days \\
\hline $\begin{array}{l}\text { Mannacio } \\
\text { et al. }{ }^{30} \\
(2008)\end{array}$ & 200 & Italy & $\begin{array}{l}\text { The Journal } \\
\text { of Thoracic } \\
\text { and Cardio- } \\
\text { vascular } \\
\text { Surgery }\end{array}$ & CABG & $\begin{array}{l}\text { Rosuvastatin } \\
(20 \mathrm{mg}) \\
\text { versus } \\
\text { Placebo }\end{array}$ & 7 days & 25 days \\
\hline $\begin{array}{l}\text { Osmanovic } \\
\text { et al. }{ }^{31} \\
(2019)\end{array}$ & 160 & Bosnia & $\begin{array}{l}\text { Medical } \\
\text { Archives }\end{array}$ & CABG & $\begin{array}{l}\text { Rosouvastatin } \\
20 \mathrm{mg} \text { versus } \\
\text { Placebo }\end{array}$ & 7-10 days & $\begin{array}{l}\text { until } \\
\text { hospital } \\
\text { discharge }\end{array}$ \\
\hline $\begin{array}{l}\text { Park et al. } \\
\mathbf{3 2}^{2}(2016)\end{array}$ & 200 & Korea & $\begin{array}{l}\text { Intensive } \\
\text { Care } \\
\text { Medicine }\end{array}$ & Multiple & $\begin{array}{l}\text { Atorvastatin } \\
\text { (variable) } \\
\text { versus } \\
\text { Placebo }\end{array}$ & $12-24 \mathrm{~h}$ & $\begin{array}{l}\text { until } \\
\text { hospital } \\
\text { discharge }\end{array}$ \\
\hline $\begin{array}{l}\text { Patti et al. } \\
{ }^{33}(2006)\end{array}$ & 200 & Italy & Circulation & Multiple & $\begin{array}{l}\text { Atorvastatin } \\
(40 \mathrm{mg}) \\
\text { versus } \\
\text { Placebo }\end{array}$ & 7 days & 30 days \\
\hline $\begin{array}{l}\text { Song et al. } \\
34 \text { (2008) }\end{array}$ & 124 & Korea & $\begin{array}{l}\text { American } \\
\text { Heart } \\
\text { Journal }\end{array}$ & CABG & $\begin{array}{l}\text { Atorvastatin } \\
(20 \mathrm{mg}) \\
\text { versus } \\
\text { Placebo }\end{array}$ & 3 days & 30 days \\
\hline $\begin{array}{l}\text { Spadaccio } \\
\text { et al. }{ }^{\mathbf{3 5}} \\
(2010)\end{array}$ & 50 & Italy & $\begin{array}{l}\text { Journal of } \\
\text { Cardiovascu- } \\
\text { lar } \\
\text { Pharmacology }\end{array}$ & CABG & $\begin{array}{l}\text { Atorvastatin } \\
(20 \mathrm{mg}) \\
\text { versus } \\
\text { Placebo }\end{array}$ & 21 days & 7 days \\
\hline $\begin{array}{l}\text { Sun et al. } \\
\mathbf{3 6}_{(2011)}\end{array}$ & 100 & China & $\begin{array}{l}\text { International } \\
\text { Heart } \\
\text { Journal }\end{array}$ & CABG & $\begin{array}{l}\text { Atorvastatin } \\
(20 \mathrm{mg}) \\
\text { versus } \\
\text { Placebo }\end{array}$ & 7 days & 14 days \\
\hline $\begin{array}{l}\text { Vukovic et } \\
\text { al. }{ }^{37} \\
(2011)\end{array}$ & 52 & Serbia & Perfusion & CABG & $\begin{array}{l}\text { Atorvastatin } \\
(20 \mathrm{mg}) \\
\text { versus } \\
\text { Placebo }\end{array}$ & 21 days & - \\
\hline
\end{tabular}




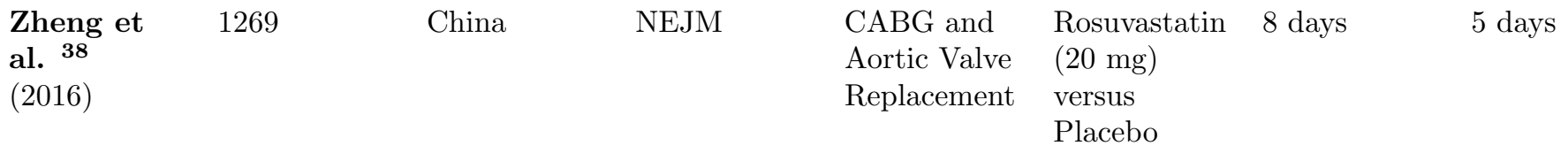

\subsection{Statin versus Placebo}

\begin{tabular}{|c|c|c|c|c|c|c|c|c|}
\hline Study or Subgroup & Statin & notal & \multicolumn{2}{|c|}{ Control } & Weight & $\begin{array}{c}\text { Odds Ratio } \\
\text { M-H, Fixed, } 95 \% \mathrm{Cl}\end{array}$ & \multicolumn{2}{|c|}{$\begin{array}{c}\text { Odds Ratio } \\
\text { M-H, Fixed, } 95 \% \mathrm{CI}\end{array}$} \\
\hline Allah 2019 & 6 & 31 & 14 & 30 & $0.0 \%$ & $0.27[0.09,0.86]$ & & \\
\hline Baran 2011 & 1 & 30 & 7 & 30 & $0.0 \%$ & $0.11[0.01,0.99]$ & & \\
\hline Billings 2016 & 43 & 102 & 28 & 97 & $7.8 \%$ & $1.80[1.00,3.24]$ & & $=$ \\
\hline Dehghani 2015 & 6 & 29 & 13 & 29 & $0.0 \%$ & $0.32[0.10,1.02]$ & & \\
\hline ji 2009 & 10 & 71 & 23 & 69 & $0.0 \%$ & $0.33[0.14,0.76]$ & & \\
\hline Mannacio 2008 & 18 & 100 & 35 & 100 & $13.5 \%$ & $0.41[0.21,0.78]$ & $\rightarrow$ & \\
\hline Osmanovic 2019 & 27 & 80 & 25 & 80 & $7.8 \%$ & $1.12[0.58,2.17]$ & & \\
\hline Park 2016 & 11 & 100 & 10 & 100 & $4.2 \%$ & $1.11[0.45,2.75]$ & - & \\
\hline Patti 2006 & 35 & 101 & 56 & 99 & $17.4 \%$ & $0.41[0.23,0.72]$ & $\rightarrow$ & \\
\hline Song 2008 & 8 & 62 & 17 & 62 & $0.0 \%$ & $0.39[0.15,0.99]$ & & \\
\hline Spadaccio 2010 & 2 & 25 & 4 & 25 & $0.0 \%$ & $0.46[0.08,2.75]$ & & \\
\hline Sun 2011 & 9 & 49 & 21 & 51 & $0.0 \%$ & $0.32[0.13,0.80]$ & & \\
\hline Vukovic 2011 & 2 & 27 & 8 & 25 & $0.0 \%$ & $0.17[0.03,0.90]$ & & \\
\hline Zheng 2016 & 134 & 639 & 132 & 630 & $49.4 \%$ & $1.00[0.76,1.31]$ & & \\
\hline Total $(95 \% \mathrm{Cl})$ & & 1122 & & 1106 & $100.0 \%$ & $0.89[0.74,1.09]$ & 4 & 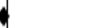 \\
\hline Total events & 268 & & 286 & & & & & \\
\hline $\begin{array}{l}\text { Heterogeneity. Chi }{ }^{2}= \\
\text { Test for overall effect }\end{array}$ & $\begin{array}{l}19.54, d \\
Z=1.13\end{array}$ & $\begin{array}{l}=5 \\
(P=0\end{array}$ & $\begin{array}{l}=0.00 \\
261\end{array}$ & & & & $\begin{array}{cc}0.002 & 0.1 \\
\text { Favours [Statin] }\end{array}$ & \begin{tabular}{|l}
1 \\
$\mid 1$ \\
$\mid$ Favours
\end{tabular} \\
\hline
\end{tabular}

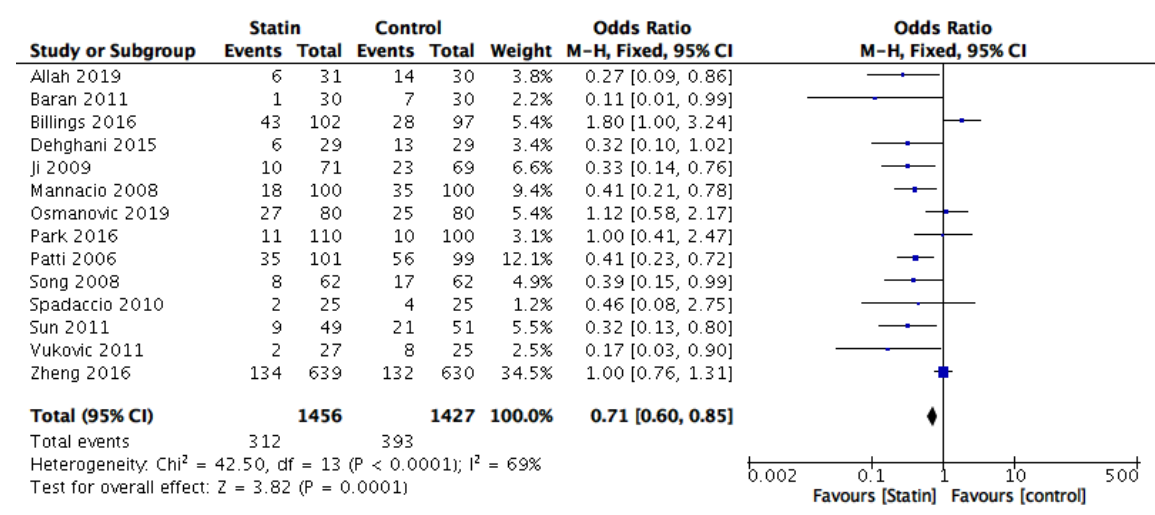

Fourteen studies comprising 2883 patients reported the POAF prevalence in patients pretreated with a statin or placebo ${ }^{\mathbf{2 5 - 3 8}}$. Statin was correlated to a reduced prevalence of POAF (312/1456 [21.4\%] versus 393/1427 $[27.5 \%]$ ). We encountered a statistically significant difference between the two considered groups (OR 0.71; $95 \%$ CI: 0.60 to 0.85, p-value $=0.0001)$. There was severe heterogeneity observed between the 14 studies $\left(\mathrm{I}^{2}=69 \%\right)$ (Figure 4$)$. However, If we only consider studies with [?] 199 patients, the results are divergent $\left(\right.$ OR $0.89 ; 95 \%$ CI: 0.74 to 1.09 , p-value $\left.=0.26 ; \mathrm{I}^{2}=74 \%\right)($ Figure 5$)$. 


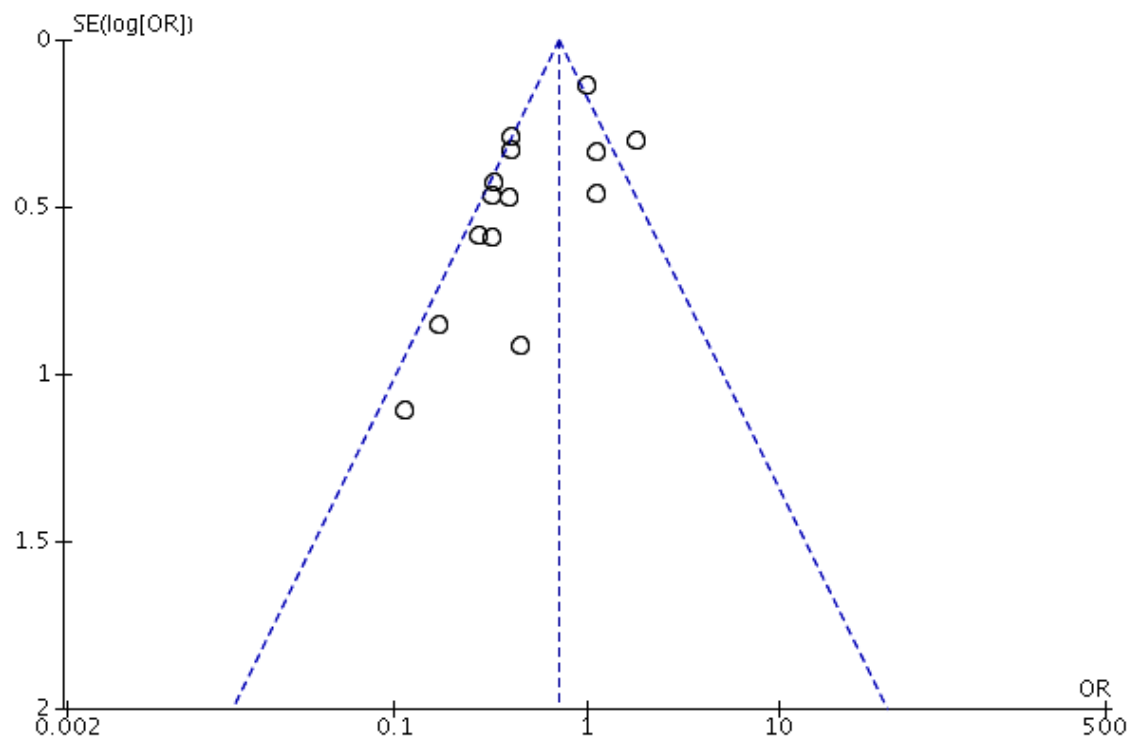

\subsection{Rosuvastatin versus Placebo}

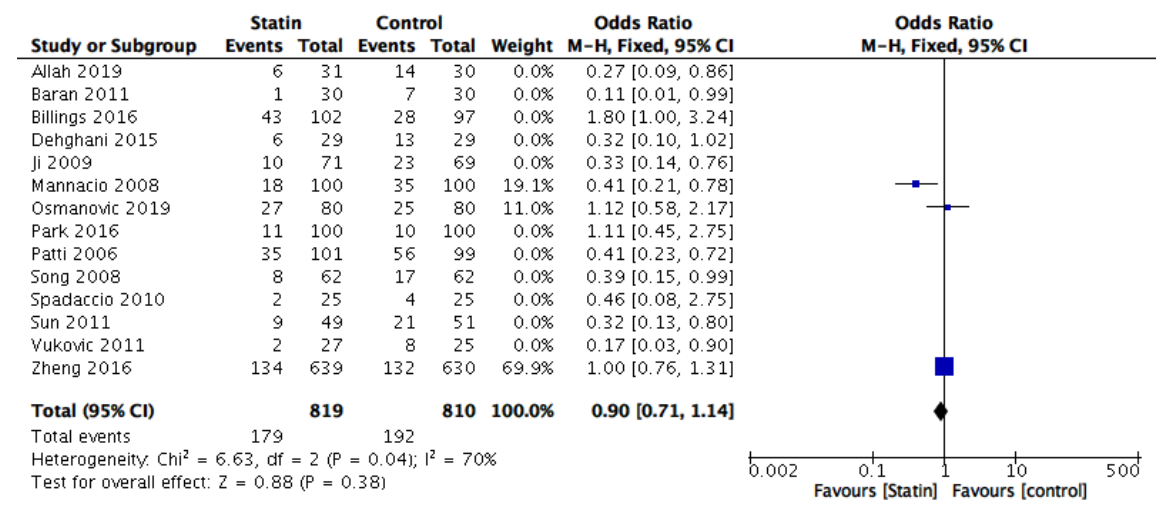

Three studies including 1629 patients reported the POAF in patients pretreated with rosuvastatin or placebo $^{\mathbf{3 0 , 3 1 , 3 8}}$. Rosuvastatin was correlated to a reduced prevalence of POAF (179/819 [21.9\%] versus $192 / 810[23.7 \%]$ ). However, there was no statistically significant difference between the two groups (OR 0.90; $95 \%$ CI: 0.71 to 1.14 , p-value $=0.38)$. There was severe heterogeneity observed between the three inherent studies $\left(\mathrm{I}^{2}=70 \%\right.$ ) (Figure 7 ). If we only consider studies with [?] 199 patients, the results are similar (OR 0.87; 95\% CI: 0.68 to 1.12 , p-value $\left.=0.29 ; \mathrm{I}^{2}=84 \%\right)($ Figure 8$)$. 


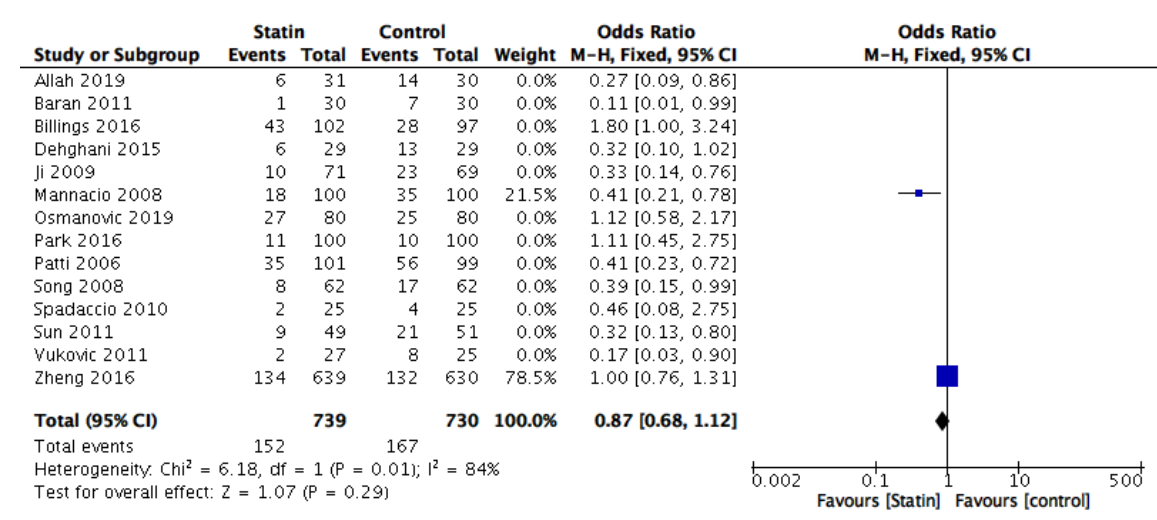

\subsection{Atorvastatin versus Placebo}

\begin{tabular}{|c|c|c|c|c|c|c|c|c|}
\hline Study or Subgroup & \multicolumn{2}{|c|}{ Statin } & Control & $\begin{array}{l}\text { rol } \\
\text { Total }\end{array}$ & Weight & \multirow{2}{*}{$\begin{array}{c}\text { Odds Ratio } \\
\text { M-H, Fixed, 95\% Cl } \\
0.27[0.09086]\end{array}$} & \multicolumn{2}{|c|}{$\begin{array}{c}\text { Odds Ratio } \\
\text { M-H, Fixed, } 95 \% \mathrm{CI}\end{array}$} \\
\hline Allah 2019 & 6 & 31 & 14 & 30 & $7.4 \%$ & & & \\
\hline Baran 2011 & 1 & 30 & 7 & 30 & $4.4 \%$ & $0.11[0.01,0.99]$ & & \\
\hline Billings 2016 & 43 & 102 & 28 & 97 & $10.8 \%$ & $1.80[1.00,3.24]$ & & $=$ \\
\hline Dehghani 2015 & 6 & 29 & 13 & 29 & $6.7 \%$ & $0.32[0.10,1.02]$ & & \\
\hline Ji 2009 & 10 & 71 & 23 & 69 & $13.0 \%$ & $0.33[0.14,0.76]$ & $\rightarrow-$ & \\
\hline Mannacio 2008 & 18 & 100 & 35 & 100 & $0.0 \%$ & $0.41[0.21,0.78]$ & & \\
\hline Osmanovic 2019 & 27 & 80 & 25 & 80 & $0.0 \%$ & $1.12[0.58,2.17]$ & & \\
\hline Park 2016 & 11 & 100 & 10 & 100 & $5.8 \%$ & $1.11[0.45,2.75]$ & - & \\
\hline Patti 2006 & 35 & 101 & 56 & 99 & $24.0 \%$ & $0.41[0.23,0.72]$ & $\rightarrow-$ & \\
\hline Song 2008 & 8 & 62 & 17 & 62 & $9.6 \%$ & $0.39[0.15,0.99]$ & & \\
\hline Spadaccio 2010 & 2 & 25 & 4 & 25 & $2.4 \%$ & $0.46[0.08,2.75]$ & & \\
\hline Sun 2011 & 9 & 49 & 21 & 51 & $10.9 \%$ & $0.32[0.13,0.80]$ & $\rightarrow$ & \\
\hline Vukovic 2011 & 2 & 27 & 8 & 25 & $5.0 \%$ & $0.17[0.03,0.90]$ & & \\
\hline Zheng 2016 & 134 & 639 & 132 & 630 & $0.0 \%$ & $1.00[0.76,1.31]$ & & \\
\hline Total $(95 \% \mathrm{Cl})$ & & 627 & & 617 & $100.0 \%$ & $0.54[0.41,0.70]$ & $\bullet$ & \\
\hline Total events & 133 & & 201 & & & & & \\
\hline $\begin{array}{l}\text { Heterogeneity. } \mathrm{Chi}^{2}= \\
\text { Test for overall effect }\end{array}$ & $\begin{array}{l}28.43, d \\
z=4.64\end{array}$ & $\begin{array}{l}=10 \\
(P<0\end{array}$ & $\begin{array}{l}(P=0.00 \\
00001)\end{array}$ & $02) ; 1^{2}=$ & & & 0.002 & \\
\hline
\end{tabular}

Eleven studies comprising a total of 1244 patients reported the POAF in patients pretreated with atorvastatin or placebo ${ }^{\mathbf{2 5 - 2 9 , 3 2 - 3 7}}$. Atorvastatin was correlated to a reduced prevalence of POAF (133/627 [21.2\%] versus $201 / 617$ [32.6\%]). There was a statistically significant difference between the considered groups (OR 0.54; $95 \%$ CI: 0.41 to 0.70 , p-value $<0,00001)$. There was severe heterogeneity observed between the 11 studies $\left(\mathrm{I}^{2}=65 \%\right)$ (Figure 9$)$. However, If we only consider studies with [?] 199 patients, the results are different $\left(\right.$ OR $0.74 ; 95 \%$ CI: 0.54 to 1.03, p-value $\left.=0.08 ; \mathrm{I}^{2}=83 \%\right)($ Figure 10$)$.

\begin{tabular}{|c|c|c|c|c|c|c|c|c|}
\hline Study or Subgroup & \multicolumn{2}{|c|}{ Statin } & \multicolumn{2}{|c|}{ Control } & Weight & $\begin{array}{c}\text { Odds Ratio } \\
\text { M-H, Fixed, } 95 \% \mathrm{Cl}\end{array}$ & \multicolumn{2}{|c|}{$\begin{array}{c}\text { Odds Ratio } \\
\text { M-H, Fixed, } 95 \% \mathrm{Cl}\end{array}$} \\
\hline Allah 2019 & 6 & 31 & 14 & 30 & $0.0 \%$ & $0.27[0.09,0.86]$ & & \\
\hline Baran 2011 & 1 & 30 & 7 & 30 & $0.0 \%$ & $0.11[0.01,0.99]$ & & \\
\hline Billings 2016 & 43 & 102 & 28 & 97 & $20.1 \%$ & $1.80[1.00,3.24]$ & & $=-$ \\
\hline Dehghani 2015 & 6 & 29 & 13 & 29 & $0.0 \%$ & $0.32[0.10,1.02]$ & & \\
\hline Ji 2009 & 10 & 71 & 23 & 69 & $24.3 \%$ & $0.33[0.14,0.76]$ & $\longrightarrow-$ & \\
\hline Mannacio 2008 & 18 & 100 & 35 & 100 & $0.0 \%$ & $0.41[0.21,0.78]$ & & \\
\hline Osmanovic 2019 & 27 & 80 & 25 & 80 & $0.0 \%$ & $1.12[0.58,2.17]$ & & \\
\hline Park 2016 & 11 & 100 & 10 & 100 & $10.8 \%$ & $1.11[0.45,2.75]$ & - & - \\
\hline Patti 2006 & 35 & 101 & 56 & 99 & $44.8 \%$ & $0.41[0.23,0.72]$ & - & \\
\hline Song 2008 & 8 & 62 & 17 & 62 & $0.0 \%$ & $0.39[0.15,0.99]$ & & \\
\hline Spadaccio 2010 & 2 & 25 & 4 & 25 & $0.0 \%$ & $0.46[0.08,2.75]$ & & \\
\hline Sun 2011 & 9 & 49 & 21 & 51 & $0.0 \%$ & $0.32[0.13,0.80]$ & & \\
\hline Vukovic 2011 & 2 & 27 & 8 & 25 & $0.0 \%$ & $0.17[0.03,0.90]$ & & \\
\hline Zheng 2016 & 134 & 639 & 132 & 630 & $0.0 \%$ & $1.00[0.76,1.31]$ & & \\
\hline Total $(95 \% \mathrm{Cl})$ & & 374 & & 365 & $100.0 \%$ & $0.74[0.54,1.03]$ & & \\
\hline Total events & 99 & & 117 & & & & & \\
\hline $\begin{array}{l}\text { Heterogeneity. } \mathrm{Chi}^{2}= \\
\text { Test for overall effect }\end{array}$ & $\begin{array}{l}17.33, d f \\
z=1.77\end{array}$ & $\begin{array}{l}=3(\mathrm{P} \\
(\mathrm{P}=0 .\end{array}$ & $\begin{array}{l}=0.000 \\
0.08\end{array}$ & $06) ;\left.\right|^{2}=$ & & & $\begin{array}{|cc|}0.002 & 0.1 \\
& \text { Favours [Statin] }\end{array}$ & $\begin{array}{l}10 \\
10 \\
\text { Favours }\end{array}$ \\
\hline
\end{tabular}

\section{DISCUSSION}


POAF is a frequent early complication of cardiac surgery ${ }^{\mathbf{2 - 7}}$. Nevertheless, it leads to increased in-hospital

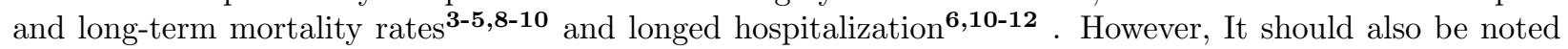
that atrial fibrillation is usually self-limited among patients suffering POAF without previous atrial arrhythmias episodes. About 15 to 30 percent convert within two hours and up to 80 percent the first 24 hours $\mathbf{2 , 3 9 , 4 0}$. Indeed, the challenge is to design cost-effective preventive therapy with impact in lowering POAF incidence.

Our review provides additional information regarding the potential association between statin and POAF reduction rate. Considering all fourteen studies, statin pre-treatment reduced the incidence of POAF compared to placebo (OR 0.71; 95\% CI: 0.60 to 0.85 , p-value $<0.00001$ ). Analyzing specific statin subclasses, atorvastatin was associated with lower incidence of POAF (OR 0.54; 95\% CI: 0.41 to 0.70, p-value $=0.002$; $\mathrm{I}^{2}=65 \%$ ), but rosuvastatin did not (OR 0.90; 95\% CI: 0.71 to 1.14 , p-value $=0.38$ ). At first analysis, it is conceivable that there is a real difference between the two subclasses of statin. However, we retain that the discrepancy in findings is due to the small number of participants and other important limitations of many trials present in the literature. Indeed, we decided to select only RCTs with [?] 199 participants. Interestingly, the results were completely conflicting. Indeed, using the above "restrictions", we found no statistically significant difference between statin pre-treatment and placebo (OR 0.89; 95\% CI: 0.74 to 1.09 , $\mathrm{p}$-value $\left.=0.26 ; \mathrm{I}^{2}=74 \%\right)$. The same can be said for atorvastatin (OR 0.74; 95\% CI: 0.54 to $1.03, \mathrm{p}$-value $=$ $0.08 ; \mathrm{I}^{2}=83 \%$ ) and rosuvastatin versus placebo (OR $0.87 ; 95 \%$ CI: 0.68 to 1.12 , p-value $=0,29 ; \mathrm{I}^{2}=84 \%$ ).

We retain that the severe heterogeneity present in our results reflects the wide distribution of POAF incidence in different studies. In fact, as previously reported, a variable number between 15 to 50 percent of patients who had valve surgery or CABG experienced POAF in the early postoperative period ${ }^{\mathbf{2 - 7}}$.

Overall, the STICS trial was the study with the fewest biases ${ }^{\mathbf{3 8}}$. Considering statin-naive patients, the authors enrolled 1269 individuals (more than one-third of the total number present in our meta-analysis). They evaluated the outcomes systematically in a blinded manner and compared outcomes between the trial arms on an intention-to-treat basis. In this study, the initiation of rosuvastatin therapy (20mg/day) before cardiac surgery did not prevent the risk for POAF $\mathbf{3 8}$. In addition, a significantly higher rate of postoperative acute kidney injury was noted (24.7 versus 19.3 percent; $\mathrm{p}=0.005)^{\mathbf{3 8}}$.

Thus, since our results match the STICS trial ones, we are confident to state that there are no differences between statin and placebo in reducing POAF. However, since high heterogeneity between studies, more randomized clinical trials with more participants are mandatory for final confirmation. The START-CABG trial is an ongoing study that will probably contribute to dispelling many doubts ${ }^{\mathbf{4 1}}$. Indeed, in this study, 2630 were randomized to receive high-dose of wide subclasses of statin or placebo given shortly before CABG. One of the valuable secondary end-points includes POAF. Thus, this trial will help to confirm or reject the current evidence.

Until then, based upon our results (different from the previous meta-analysis) $\mathbf{1 8 , 4 2}$ and possible kidney issues, we suggest avoiding statin pre-treatment in preventing POAF for statin-naive patients undergoing cardiac surgery.

\section{STUDY LIMITATIONS}

Limitations of our meta-analysis are:

heterogeneity in statin dosage;

different starting time before surgery;

different follow-up between trials;

relevant outcome heterogeneity.

\section{CONCLUSION}


Our meta-analysis suggests that statin pre-treatment before cardiac surgery is not associated with a significant reduction of POAF occurrence. Severe heterogeneity between the considered study does exist. Until the results of the START-CABG trial are available and considering the potential acute kidney injury, we discourage statin pre-treatment in preventing POAF.

\section{REFERENCES}

LaPar DJ, Crosby IK, Rich JB, et al. A contemporary cost analysis of postoperative morbidity after coronary artery by- pass grafting with and without concomitant aortic valve replacement to improve patient quality and cost-effective care . Ann Thorac Surg 2013;96:1621-7;

Maisel WH, Rawn JD, Stevenson WG. Atrial fibrillation after cardiac surgery. Ann Intern Med. 2001;135(12):1061-1073. doi:10.7326/0003-4819-135-12-200112180-00010;

Mathew JP, Fontes ML, Tudor IC, et al. A multicenter risk index for atrial fibrillation after cardiac surgery. JAMA. 2004;291(14):1720-1729. doi:10.1001/jama.291.14.1720;

Villareal RP, Hariharan R, Liu BC, et al. Postoperative atrial fibrillation and mortality after coronary artery bypass surgery. J Am Coll Cardiol. 2004;43(5):742-748. doi:10.1016/j.jacc.2003.11.023;

Mariscalco G, Klersy C, Zanobini M, et al. Atrial fibrillation after isolated coronary surgery affects late survival. Circulation. 2008;118(16):1612-1618. doi:10.1161/CIRCULATIONAHA.108.777789;

Creswell LL, Schuessler RB, Rosenbloom M, Cox JL. Hazards of postoperative atrial arrhythmias. Ann Thorac Surg. 1993;56(3):539-549. doi:10.1016/0003-4975(93)90894-n;

Asher CR, Miller DP, Grimm RA, Cosgrove DM 3rd, Chung MK.Analysis of risk factors for development of atrial fibrillation early after cardiac valvular surgery. Am J Cardiol. 1998;82(7):892-895. doi:10.1016/s0002-9149(98)00498-6;

Bramer S, van Straten AH, Soliman Hamad MA, Berreklouw E, Martens EJ, Maessen JG. The impact of new-onset postoperative atrial fibrillation on mortality after coronary artery bypass grafting. Ann Thorac Surg. 2010;90(2):443-449. doi:10.1016/j.athoracsur.2010.03.083;

Saxena A, Dinh DT, Smith JA, Shardey GC, Reid CM, Newcomb AE.Usefulness of postoperative atrial fibrillation as an independent predictor for worse early and late outcomes after isolated coronary artery bypass grafting (multicenter Australian study of 19,497 patients). Am J Cardiol. 2012;109(2):219-225. doi:10.1016/j.amjcard.2011.08.033;

Kosmidou I, Chen S, Kappetein AP, et al. New-Onset Atrial Fibrillation After PCI or CABG for Left Main Disease: The EXCEL Trial.J Am Coll Cardiol. 2018;71(7):739-748. doi:10.1016/j.jacc.2017.12.012;

Aranki SF, Shaw DP, Adams DH, et al. Predictors of atrial fibrillation after coronary artery surgery. Current trends and impact on hospital resources. Circulation. 1996;94(3):390-397. doi:10.1161/01.cir.94.3.390;

Crystal E, Garfinkle MS, Connolly SS, Ginger TT, Sleik K, Yusuf SS.Interventions for preventing postoperative atrial fibrillation in patients undergoing heart surgery. Cochrane Database Syst Rev. 2004;(4):CD003611. Published 2004 Oct 18. doi:10.1002/14651858.CD003611.pub2;

Maesen B, Nijs J, Maessen J, Allessie M, Schotten U.Post-operative atrial fibrillation: a maze of mechanisms.Europace 2012;14:159-74;

Pinho-Gomes AC, Reilly S, Brandes RP, Casadei B. Targeting inflammation and oxidative stress in atrial fibrillation: role of 3-hydroxy-3-methylglutaryl-coenzyme a reductase inhibition with statins. Antioxid Redox Signal 2014;20:1268-85; 
Antoniades C, Demosthenous M, Reilly S, et al. Myocardial redox state pre- dicts in-hospital clinical outcome after cardiac surgery effects of short-term pre- operative statin treatment. J Am Coll Cardiol 2012;59:60-70;

Antoniades C, Bakogiannis C, Leeson P, et al. Rapid, direct effects of statin treatment on arterial redox state and nitric oxide bioavailability in human atherosclerosis via tetrahydrobiopterinmediated endothelial nitric oxide synthase coupling. Circulation 2011;124:335-45;

Kostapanos MS, Liberopoulos EN, Goudevenos JA, et al. Do statins have an anti arrhythmic activity? Cardiovasc Res 2007; 75:10 - 20;

Fauchier L, Clementy N, Babuty D. Statin therapy and atrial fibrillation: systematic review and updated meta-analysis of published randomized controlled trials. Curr Opin Cardiol. 2013;28(1):718. doi:10.1097/HCO.0b013e32835b0956;

Kuhn EW, Liakopoulos OJ, Stange S, et al. Preoperative statin therapy in cardiac surgery: a meta-analysis of 90,000 patients. Eur J Cardiothorac Surg 2014;45:17-26;

Moher D, Liberati A, Tetzlaff J, Altman DG; PRISMA Group.Preferred reporting items for systematic reviews and meta-analyses: the PRISMA statement. PLoS Med . 2009;6(7):e1000097. doi:10.1371/journal.pmed.1000097;

Landis JR, Koch GG. The measurement of observer agreement for categorical data. Biometrics . 1977;33(1):159-174;

Higgins J, Altman D, Sterne. Chapter 8: Assessing risk of bias in included studies. In: Higgins J, Green S, eds. Cochrane Handbook for Systematic Reviews of Interventions 5.1.0 ed. The Cochrane Collaboration. https://handbook-5-1.cochrane.org/ 2011;

Higgins JP, Thompson SG. Quantifying heterogeneity in a meta-analysis. Stat Med . 2002;21(11):1539-1558. doi:10.1002/sim.1186;

Higgins JP, Thompson SG, Deeks JJ, Altman DG. Measuring inconsistency in meta-analyses. BMJ . 2003;327(7414):557-560. doi:10.1136/bmj.327.7414.557;

Allah EA, Kamel EZ, Osman HM, et al. Could Short-Term Perioperative High-Dose Atorvastatin Offer Antiarrhythmic and Cardio-Protective Effects in Rheumatic Valve Replacement Surgery?. J Cardiothorac Vasc Anesth. 2019;33(12):3340-3347. doi:10.1053/j.jvca.2019.05.013;

Baran Ç, Durdu S, Dalva K, et al. Effects of preoperative short term use of atorvastatin on endothelial progenitor cells after coronary surgery: a randomized, controlled trial. Stem Cell Rev Rep. 2012;8(3):963-971. doi:10.1007/s12015-011-9321-z;

Billings FT 4th, Hendricks PA, Schildcrout JS, et al. High-Dose Perioperative Atorvastatin and Acute Kidney Injury Following Cardiac Surgery: A Randomized Clinical Trial. JAMA. 2016;315(9):877888. doi:10.1001/jama.2016.0548

Dehghani MR, Kasianzadeh M, Rezaei Y, Sepehrvand N. Atorvastatin Reduces the Incidence of Postoperative Atrial Fibrillation in Statin-Naive Patients Undergoing Isolated Heart Valve Surgery: A Double-Blind, Placebo-Controlled Randomized Trial. J Cardiovasc Pharmacol Ther. 2015;20(5):465-472. doi:10.1177/1074248414564869;

Ji Q, Mei Y, Wang X, et al. Effect of preoperative atorvastatin therapy on atrial fibrillation following off-pump coronary artery bypass grafting . Circ J. 2009;73(12):2244-2249. doi:10.1253/circj.cj09-0352:

Mannacio VA, Iorio D, De Amicis V, Di Lello F, Musumeci F.Effect of rosuvastatin pretreatment on myocardial damage after coronary surgery: a randomized trial. J Thorac Cardiovasc Surg. 2008;136(6):1541-1548. doi:10.1016/j.jtcvs.2008.06.038 
Osmanovic E, Ostojic M, Avdic S, et al. Pharmacological Prophylaxis of Atrial Fibrillation After Surgical Myocardial Revascularization. Med Arch. 2019;73(1):19-22. doi:10.5455/medarh.2019.73.1922 ;

Park JH, Shim JK, Song JW, Soh S, Kwak YL. Effect of atorvastatin on the incidence of acute kidney injury following valvular heart surgery: a randomized, placebo-controlled trial. Intensive Care Med. 2016;42(9):1398-1407. doi:10.1007/s00134-016-4358-8

Patti G, Chello M, Candura D, et al. Randomized trial of atorvastatin for reduction of postoperative atrial fibrillation in patients undergoing cardiac surgery: results of the ARMYDA-3 (Atorvastatin for Reduction of MYocardial Dysrhythmia After cardiac surgery) study. Circulation. 2006;114(14):1455-1461. doi:10.1161/CIRCULATIONAHA.106.621763;

Song YB, On YK, Kim JH, et al. The effects of atorvastatin on the occurrence of postoperative atrial fibrillation after off-pump coronary artery bypass grafting surgery. Am Heart J. 2008;156(2):373.e9-373.e3.73E16. doi:10.1016/j.ahj.2008.04.020;

Spadaccio C, Pollari F, Casacalenda A, et al. Atorvastatin increases the number of endothelial progenitor cells after cardiac surgery: a randomized control study. J Cardiovasc Pharmacol. 2010;55(1):3038. doi:10.1097/FJC.0b013e3181c37d4d

Sun Y, Ji Q, Mei Y, et al. Role of preoperative atorvastatin administration in protection against postoperative atrial fibrillation following conventional coronary artery bypass grafting. Int Heart J. 2011;52(1):7-11. doi:10.1536/ihj.52.7;

Vukovic PM, Maravic-Stojkovic VR, Peric MS, et al. Steroids and statins: an old and a new antiinflammatory strategy compared.Perfusion. 2011;26(1):31-37. doi:10.1177/0267659110385607;

Zheng Z, Jayaram R, Jiang L, et al. Perioperative Rosuvastatin in Cardiac Surgery. N Engl J Med. 2016;374(18):1744-1753. doi:10.1056/NEJMoa1507750;

Soucier RJ, Mirza S, Abordo MG, et al. Predictors of conversion of atrial fibrillation after cardiac operation in the absence of class I or III antiarrhythmic medications. Ann Thorac Surg. 2001;72(3):694-698. doi:10.1016/s0003-4975(01)02817-x;

Lee JK, Klein GJ, Krahn AD, et al. Rate-control versus conversion strategy in postoperative atrial fibrillation: a prospective, randomized pilot study . Am Heart J. 2000;140(6):871-877. doi:10.1067/mhj.2000.111104;

Liakopoulos OJ, Kuhn EW, Hellmich M, et al. Statin Recapture Therapy before Coronary Artery Bypass Grafting Trial: Rationale and study design of a multicenter, randomized, double-blinded controlled clinical trial. Am Heart J. 2015;170(1):46-54.e542. doi:10.1016/j.ahj.2015.04.015;

Kuhn EW, Slottosch I, Wahlers T, Liakopoulos OJ. Preoperative statin therapy for patients undergoing cardiac surgery. Cochrane Database Syst Rev 2015;8: CD008493. 